\title{
Proof of concept of an imaging system demonstrator for PET applications with SiPM
}

\author{
Matteo Morrocchi , Sara Marcatili , Nicola Belcari , Maria Giuseppina Bisogni , \\ Gianmaria Collazuol , Giovanni Ambrosi , Cristiano Santoni . Francesco Corsi , \\ Maurizio Foresta , Cristoforo Marzocca , Gianvito Matarrese , Giancarlo Sportelli , \\ Pedro Guerra , Andres Santos ', Alberto Del Guerra
}

\begin{abstract}
A B S T R A C T
A PET imaging system demonstrator based on LYSO crystal arrays coupled to SiPM matrices is under construction at the University and INFN of Pisa. Two SiPM matrices, composed of $8 \times 8$ SiPM pixels, and $1,5 \mathrm{~mm}$ pitch, have been coupled one to one to a LYSO crystals array and read out by a custom electronics system. front-end ASICs were used to read 8 channels of each matrix. Data from each frontend were multiplexed and sent to a DAQ board for the digital conversion; a motherboard collects the data and communicates with a host computer through a USB port for the storage and off-line data processing. In this paper we show the first preliminary tomographic image of a point-like radioactive source acquired with part of the two detection heads in time coincidence.
\end{abstract}

\section{Introduction}

Positron emission tomography is a clinical diagnostic imaging technique that provides the tri-dimensional density distribution of a radiotracer injected in the body. The radiotracer is usually based on a complex molecule in which one of the atoms is a $\beta^{+}$-emitting isotope.

The two $511 \mathrm{keV}$ photons, produced by the annihilation of the positron with an electron of the absorbing medium, are detected in time coincidence by a detector ring positioned around the body. Typical PET detectors are composed of a pixellated scintillator crystal coupled to photomultipliers tubes (PMT) [1].

Silicon photomultipliers (SiPM) represent an effective alternative to PMT used in actual PET scanners. These devices are solid state detectors composed of a matrix of microcells connected in parallel; each cell is a photodiode working in Geiger mode. The total signal generated by the device is the sum of the signals produced by each fired microcell. SiPM detectors have a very fast rise time, due to the Geiger mechanism of discharge [2], are insensitive to magnetic fields [3] and show high gain $\left(10^{6}-10^{7}\right)$ at few tens of bias voltage.
Silicon-based technology allows building monolithic devices made of bi-dimensional arrays SiPMs disposed at millimetric pitch. This feature is particularly attractive in small animal PET imaging since it allows overcoming the limit in spatial resolution imposed by the current devices. Previous works showed that a SiPM based detector can reach a submillimetric spatial resolution [4], demonstrating the potentiality of this module for preclinical PET imaging.

The fast responses of the SiPMs make them suitable for Time of Flight (TOF)-PET applications aiming to improve the signal to noise ratio in the reconstructed PET image.

Furthermore, the magnetic insensitivity of the device allows the construction of hybrid PET-MRI systems that merge anatomical and functional imaging information.

We present here a PET demonstrator composed of two SiPM matrices coupled to LYSO crystal arrays and read-out by custom electronics [5].

\section{Materials and methods}

The detection module is composed of an array of $8 \times 8$ LYSO crystals, $1.5 \times 1.5 \times 10 \mathrm{~mm}^{3}$ each coupled one-to-one to a SiPM matrix. The SiPM matrix has been produced by FBK-Irst for the INFN DaSiPM2 collaboration. It is built on a common Si substrate and is fed by a common bias voltage. 


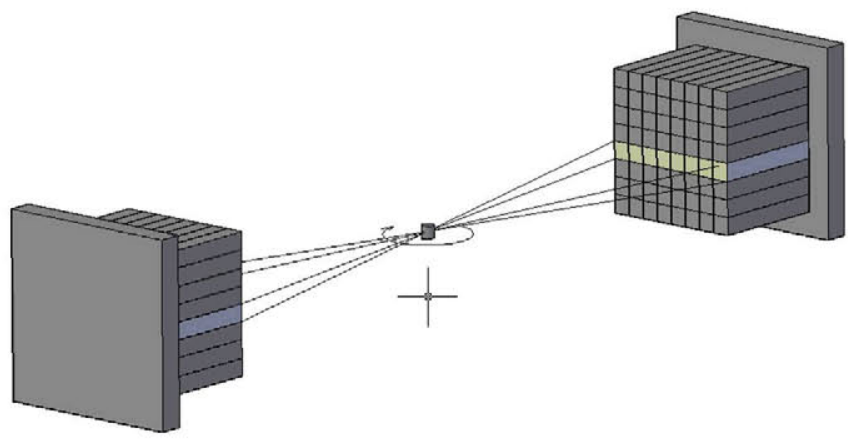

Fig. 1. Pictorial view of the acquisition set-up.

The front-end ASIC, named BASIC, has been developed by the INFN of Bari-Politecnico of Bari [6]. The ASIC is composed of 8 read-out channels, each one connected to a current buffer with a bandwith of about $350 \mathrm{MHz}$.

A current discriminator circuit located at the output of the current buffer is used to obtain a fast trigger with 16 differents threshold levels selectable.

A Charge Sensitive Amplifier (CSA) with three possible gain levels integrates the signals of each channel and the outputs of the 8 channels are multiplexed and digitized by an ADC located on a custom designed DAQ board. The digital signals provided by the DAQ boards are collected and comunicated to a PC by an FPGA Altera Stratix III located in a central motherboard [7,8].

The acquisition system is designed to handle up to 64 versus 64 channels operated in time coincidence. At the moment we are able to test only two DAQ boards (means 8 versus 8 channels). For this reason we decided to read out the central rows of two faced detection modules. A tomographic image of a ${ }^{22} \mathrm{Na}$ source has been reconstructed by placing the two modules at a distance of $120 \mathrm{~mm}$ ( $110 \mathrm{~mm}$, if we consider the center of the crystals) and operating them in time coincidence. For this test, the ${ }^{22} \mathrm{Na}$ source (nominal diameter of $1 \mathrm{~mm}$ ) has been positioned on a rotation stage at the center of the Field Of View of two detectors rows, as shown in Fig. 1.

\section{Measurements}

To obtain the tomographic image, the source has been rotated by $180^{\circ}$ at a $10^{\circ}$ step. A backprojection algorithm has been used to reconstruct the image; the obtained transaxial view of the source is depicted in Fig. 2 (an arbitrary gray level scale is shown on the right side).

The source profile (Fig. 3) presents an FWHM of about $3 \mathrm{~mm}$, if we consider a pedestal with the intensity of the mean background far from the source in the image.

The results obtained have to be considered preliminary for a number of reasons. The experimental set-up is not optimized to image. The low activity of the source (about $40 \mathrm{kBq}$ ) and the limited geometrical acceptance of the detectors imposed long acquisition times ( 2 min per view, about $40 \mathrm{~min}$ for the total datataking). Another constraint is posed by the temperature fluctuations during the acquisition time that affect the SiPM gain, resulting in an image degradation. Furthemore the reconstruction algorithm must be improved. We expect to minimize artifacts and improve the image quality by increasing the number of projections. This can be achieved by using more active sources and increasing the solid angle coverage (at the moment only one row of each detector has been read out). The stability of the SiPM gain can be improved by applying temperature control systems. Finally filtered backprojection algorithms can be used.

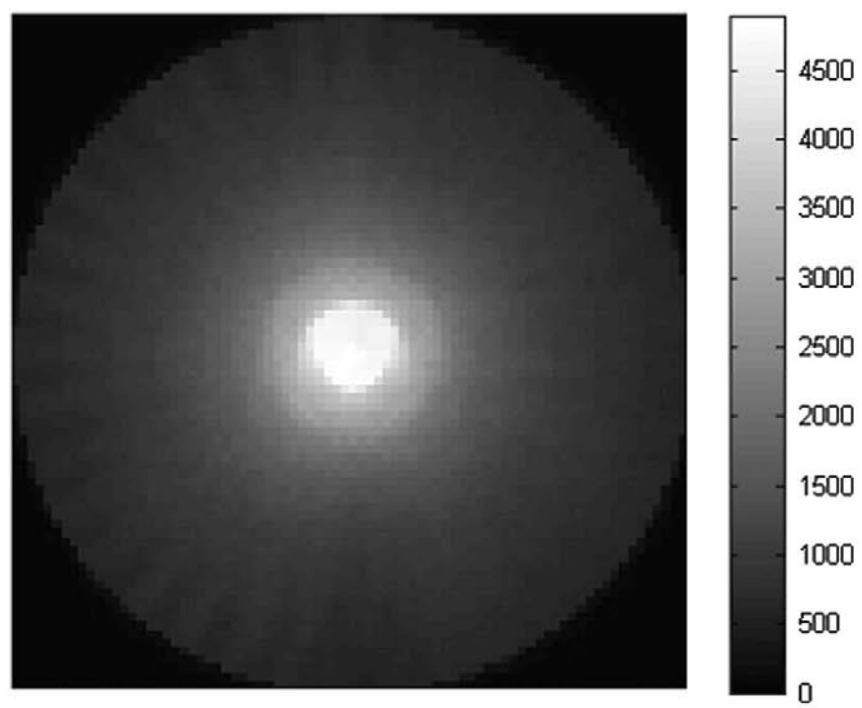

Fig. 2. Tomographic image of the ${ }^{22} \mathrm{Na}$ source reconstructed with a backprojection algorithm.

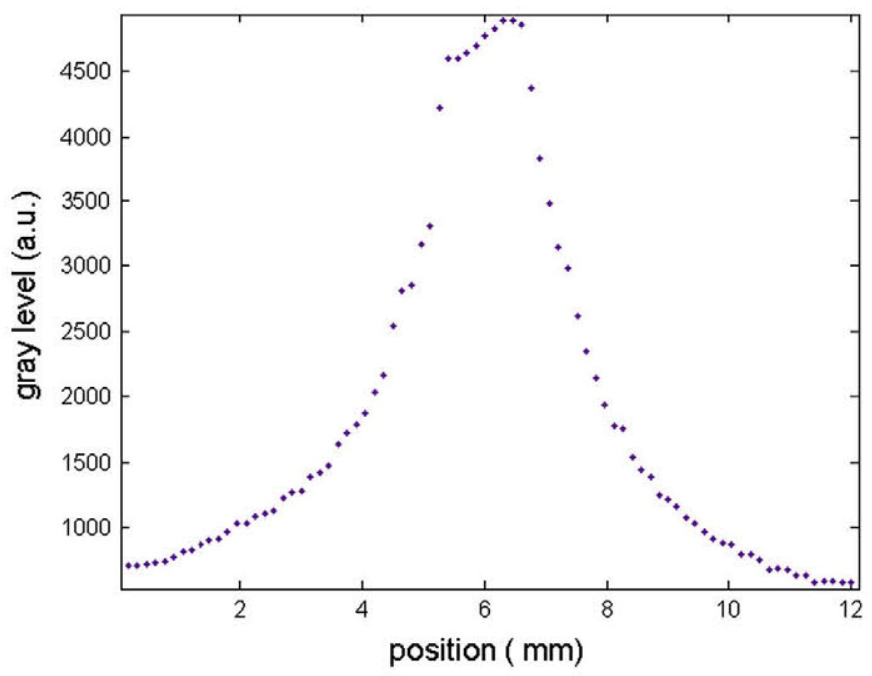

Fig. 3. Profile of the reconstructed image.

\section{Conclusions}

The tomographic image of a ${ }^{22} \mathrm{Na}$ source has been shown by using a PET demonstrator composed of two SiPM detection modules. The source has been positioned at the center of the Field of View and the transaxial view of the spot has been reconstructed. The FWHM of the central profile is about $3 \mathrm{~mm}$.

The results must be considered as preliminary since the system and the set-up were not optimized to image. Nevertheless they can serve as proof of concept that a PET system based on SiPM and integrated electronics read out is viable.

The completion of the acquisition system and the optimization of the set-up and the reconstruction algorithm will allow full exploitation of the potentiality of the SiPM technology applied to PET.

\section{Acknowledgments}

This work is partially supported by FP7-Envision project funded by EU Seven Framework. G. Sportelli, P. Guerra and A. Santos want to acknowledge partial financial support from 
Spain's Science Ministry (Projects CDTI AMIT and TEC201128972-C02-02) and European Regional Development Funds.

\section{References}

[1] W.W. Moses, Nuclear Instruments and Methods in Physics Research A 610 (2009) 11.

[2] G. Collazuol, et al., Nuclear Instruments and Methods in Physics Research A 581 (2007) 461.

[3] R.C..Hawkes,et al., IEEE Nuclear Science Symposium Conference Record-MIC, Honolulu, USA, October 28-November 3, 2007, M18-118.
[4] G. Llosà, N. Belcari, M.G. Bisogni, S. Marcatili, G. Collazuol, M. Melchiorri, C. Piemonte, P. Barrillon, S. Bondil-Blin, N. Dinu, C. deLaTaille, A. DelGuerra, Physics in Medicine and Biology 55 (2010) 7299-7315.

[5] S. Marcatili, et al., Nuclear Instruments and Methods in Physics Research A 659 (2011) 494.

[6] F. Corsi et al., BASIC: an 8-channel front-end ASIC for silicon photomultiplier detectors, N19-2, IEEE Nuclear Science Symposium Conference Record, 2009 pp. $1082-1087$.

[7] G. Sportelli, et al., IEEE Transaction on Nuclear Science NS-3 (2011) 695

[8] G. Sportelli et al., A flexible acquisition system for modular dual head positron emission tomography, IEEE Nuclear Science Symposium Conference Record (NSS/MIC), 2009, pp.3395-3398. 